\title{
SENSORY NERVE ACTION POTENTIALS IN PATIENTS WITH PERIPHERAL NERVE LESIONS
}

\author{
BY \\ R. W. GILLIATT and T. A. SEARS \\ From the Department of Applied Electrophysiology, the National Hospital, Queen Square, London
}

In 1949 Dawson and Scott described a method of examining sensory impulses in human peripheral nerves. By their technique, the median or ulnar nerve at the level of the wrist was stimulated electrically using single shocks, and the action potential of the afferent volley recorded through surface electrodes placed over the nerve trunk at a higher level in the arm. The afferent volley contained both sensory impulses and antidromic impulses in motor fibres and gave rise to a small diphasic or triphasic potential with an amplitude of less than 50 microvolts and a duration of 2 to 3 milliseconds. To eliminate the contribution of motor fibres, Dawson (1956) modified his original technique and stimulated the digital nerves through small ring electrodes wrapped round the fingers, the afferent volley being recorded as before through surface electrodes placed over the median or ulnar nerve trunk in the arm. The sensory potentials recorded were similar in form to the mixed nerve action potentials obtained previously, but, being derived from digital nerves, they were considerably smaller in amplitude.

In view of Dawson's findings it seemed worthwhile to investigate the behaviour of sensory action potentials in patients with peripheral nerve lesions, and the present paper is based on some 50 cases examined in this way. In general the technique has proved to be of considerable value in the clinical assessment of patients, and in certain cases it has provided more information about sensory nerve function than could be obtained by formal sensory testing.

\section{Methods}

These differed only in detail from those described previously by Dawson and Scott (1949) and by Dawson (1956). For stimulating the digital nerves, silver strips 2 to $4 \mathrm{~mm}$. wide were used, covered by lint moistened in saline and firmly wrapped round the finger, with the cathode placed near the metacarpo-phalangeal joint and the anode in the region of the terminal interphalangeal joint. The stimulus itself was a brief condenser discharge (time constant approximately
70 microseconds) delivered through an isolating transformer. Surface recording electrodes were of the "saddle" pattern described by Dawson and Scott (see their Fig. 1) with an inter-electrode distance of $3 \mathrm{~cm}$. Before applying the recording electrodes, the course of the median or ulnar nerve in the arm was usually mapped out by stimulation and observation of the motor response. In the few patients without any motor response to nerve stimulation the course of the nerve could not be determined in this way; in such a case recording electrodes were placed over the expected position of the nerve trunk and adjusted to give the largest response to sensory stimulation. The design of the recording electrodes was such that small lateral movements had little or no effect on the size of the recorded potential; they were thus particularly suitable for rapid tests on patients.

Nerve action potentials were amplified in the conventional way and displayed on a cathode ray tube. For photography, the method of superimposition used by Dawson (1947) was adopted; with the sweep of the cathode ray tube locked to the stimulator, records were obtained by photographing 20 successive sweeps at onesecond intervals, all 20 being superimposed on the same piece of film. This has the effect of emphasizing potentials occurring at a fixed time in the sweep, random potentials merely producing a thick base line from which even small nerve potentials can be distinguished.
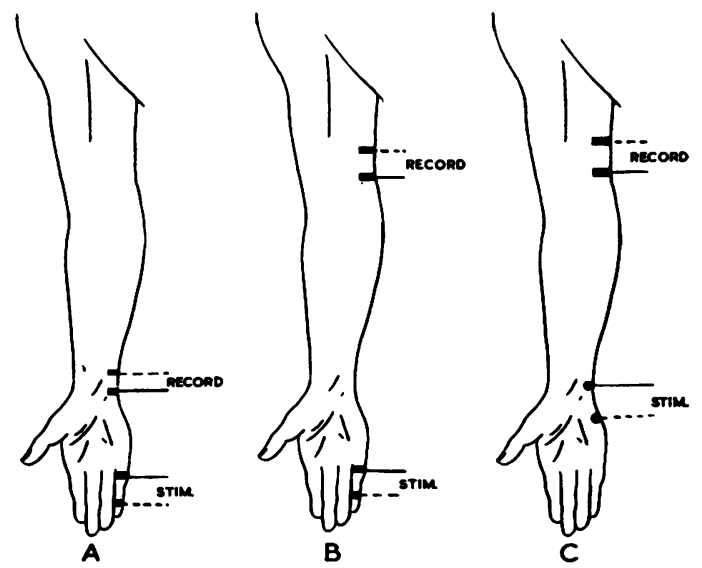

Fig. 1.-Positions used for stimulating and recording electrodes. 
When stimulating the fingers the two sites for recording electrodes used by Dawson were at the level of the wrist (Fig. 1A) and just above the elbow (Fig. 1B). In our own experience sensory action potentials recorded from the elbow region were always small and in some obese but otherwise normal subjects they were absent altogether. For this reason recording at wrist level or just above it was preferred as a standard arrangement, the stimulus being applied to the index finger when testing the median nerve and to the fifth finger for the ulnar nerve. In a few patients stimulation of the median or ulnar nerve trunk at the wrist was used with recording electrodes just above the elbow (Fig. 1C); with this arrangement the nerve action potential is large and easy to record, and although it is made up of antidromic motor as well as sensory impulses it may provide useful supplementary information.

All tests were carried out in a warm room with the subject lying on a couch and covered with blankets. Before examining people with cold hands, the arms were immersed in hot water for five or $\mathbf{1 0}$ minutes before the session, but no other method of controlling temperature was attempted.
No children were examined, and since nerve conduction velocity is known to decrease in the elderly (Wagman and Lesse, 1952), patients over the age of 70 were excluded from the present series.

Full relaxation of the subject during a test was essential as records were easily spoilt by persistent muscular contraction, motor unit action potentials being 10 or 100 times as large as the nerve potential. With patience, relaxation could usually be obtained but on a few occasions when examining apprehensive or uncooperative subjects it was necessary to give an intravenous barbiturate to cover the procedure.

\section{Results}

Control Observations on Healthy Nerves.-Sensory nerve action potentials recorded from the wrist in a healthy subject are shown in Fig. 2. In this case the triphasic form of the action potential is clearly shown, the most prominent feature being the second (upward) deflection during which the lower recording electrode becomes negative with respect to the upper one. When recording small potentials this

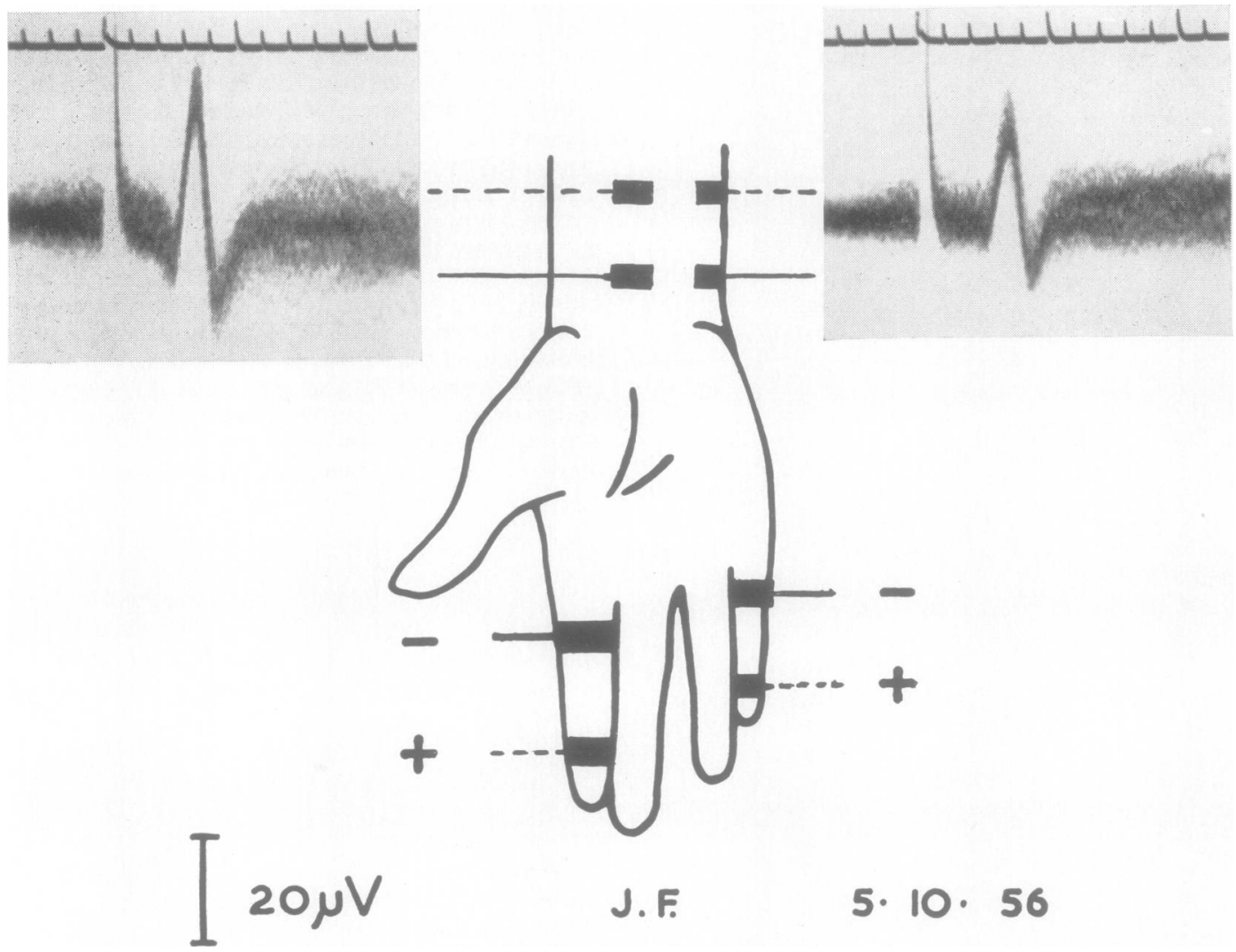

FIG. 2.-Normal sensory action potentials recorded from the median and ulnar nerve trunks at the wrist in a healthy subject. Stimuli to index and little fingers. Tracings show stimulus artefact followed by triphasic action potential. Time scale 1 and 5 milliseconds; 20 sweeps superimposed. 


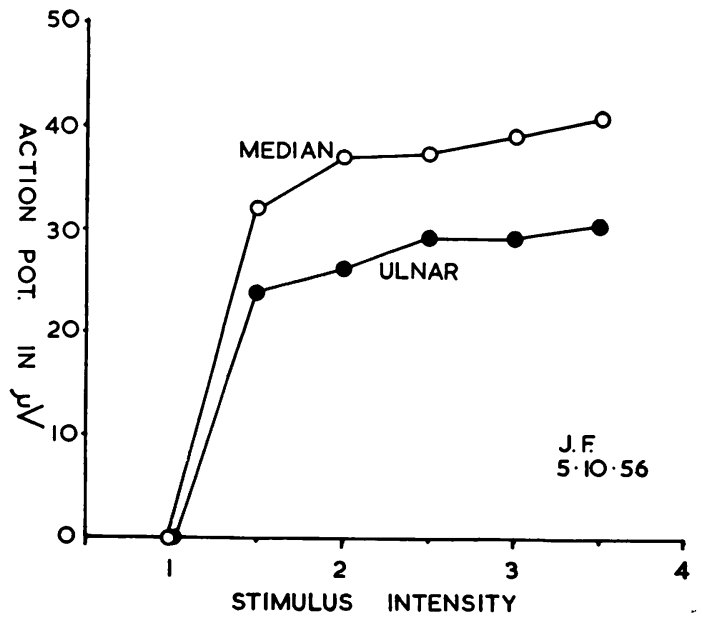

Fig. 3.-From the same experiment as in Fig. 2 to show relation between stimulus intensity and the size of sensory potentials recorded. Stimulating voltages expressed as multiples of threshold value.

triphasic form may be lost, the upward deflection still appearing but the other phases being scarcely discernible in superimposed records.

The relation between stimulus intensity and the size of the nerve response is shown in Fig. 3, from which it can be seen that the nerve action potential appears rather abruptly with increasing stimulus strength, and that above a certain critical stimulus intensity there is little further increase in action potential amplitude. With most of our normal subjects it was possible to use a stimulus intensity above this critical value, so that a further increase produced little change in the recorded potential. Strong shocks applied to the fingers produce considerable local pain, so that maximal stimuli cannot be attempted, but from what has been said above, it may be assumed that most of the large myelinated fibres in the digital nerves were being stimulated in each case. A few subjects were unable to tolerate the repetitive stimuli required to obtain superimposed records and in such cases single sweeps were photographed.

With stimuli approaching as closely as possible the maximum that could be tolerated by each subject, the size of the recorded nerve action potentials varied considerably in different individuals. For this reason a large number of healthy nerves were examined to provide a range of normal values. These control observations were made not only on healthy subjects working in the laboratory but also on the unaffected nerves of patients presenting isolated peripheral nerve lesions. In all, 28 median and 39 ulnar nerves were examined as controls.
Results of the control measurements are shown in Fig. 4, from which it can be seen that the potentials recorded from the median nerve at the wrist varied in peak-to-peak amplitude from 9 to 45 microvolts, whereas those recorded from the ulnar nerve at the wrist were generally rather smaller with a range of 8 to 28 microvolts.

For each subject the latency of the nerve action potential was measured; owing to the variability of the initial phase of the action potential, latencies were measured from the stimulus artefact to the peak of the main (negative) deflection, and in Fig. 5 this figure has been plotted for each case against the conduction distance from stimulating cathode to the lower recording electrode. For the median nerve, action potential latency varied from 2.5 to 4 milliseconds. Action potential latencies for the ulnar nerve were generally slightly shorter than for the median (range 2.2 to 3.4 milliseconds), but when the figures are corrected for conduction distance the apparent difference between the two nerves disappears.

Median Nerve Lesions at the Wrist.-Twenty-nine patients were examined with suspected median nerve compression in the carpal tunnel. These were patients referred by members of the hospital staff for routine electrodiagnosis between January, 1956, and May, 1957, and as there was no selection of cases on our part, the group as a whole contained a proportion of doubtful and atypical cases. Wasting of the thenar muscles was recorded in the hospital notes of five patients and muscular weakness without wasting in a further three. Sensory impairment, which usually took the form of mild subjective loss of superficial sensation in the fingers, had been noted in 14 cases.

When the index finger was stimulated electrically, it was noticeable that some of the patients would tolerate a higher stimulating voltage than the control group. Since we were attempting to produce a sensory action potential whenever possible, stimulus intensity was kept as high as could be tolerated by each individual but, in spite of this, the action potential recorded from the median nerve just above the wrist was frequently reduced in size. Action potential amplitude (peak-to-peak) in each case is shown in Fig. 4, from which it can be seen that the nerve action potential was absent altogether in six cases and of small amplitude in many others. Latency to the peak of the main deflection of the action potential was measurable in 23 cases, and Fig. 5 shows that it was often increased, although conduction distances were generally similar to those in the control group.

A detailed correlation between electrical and 


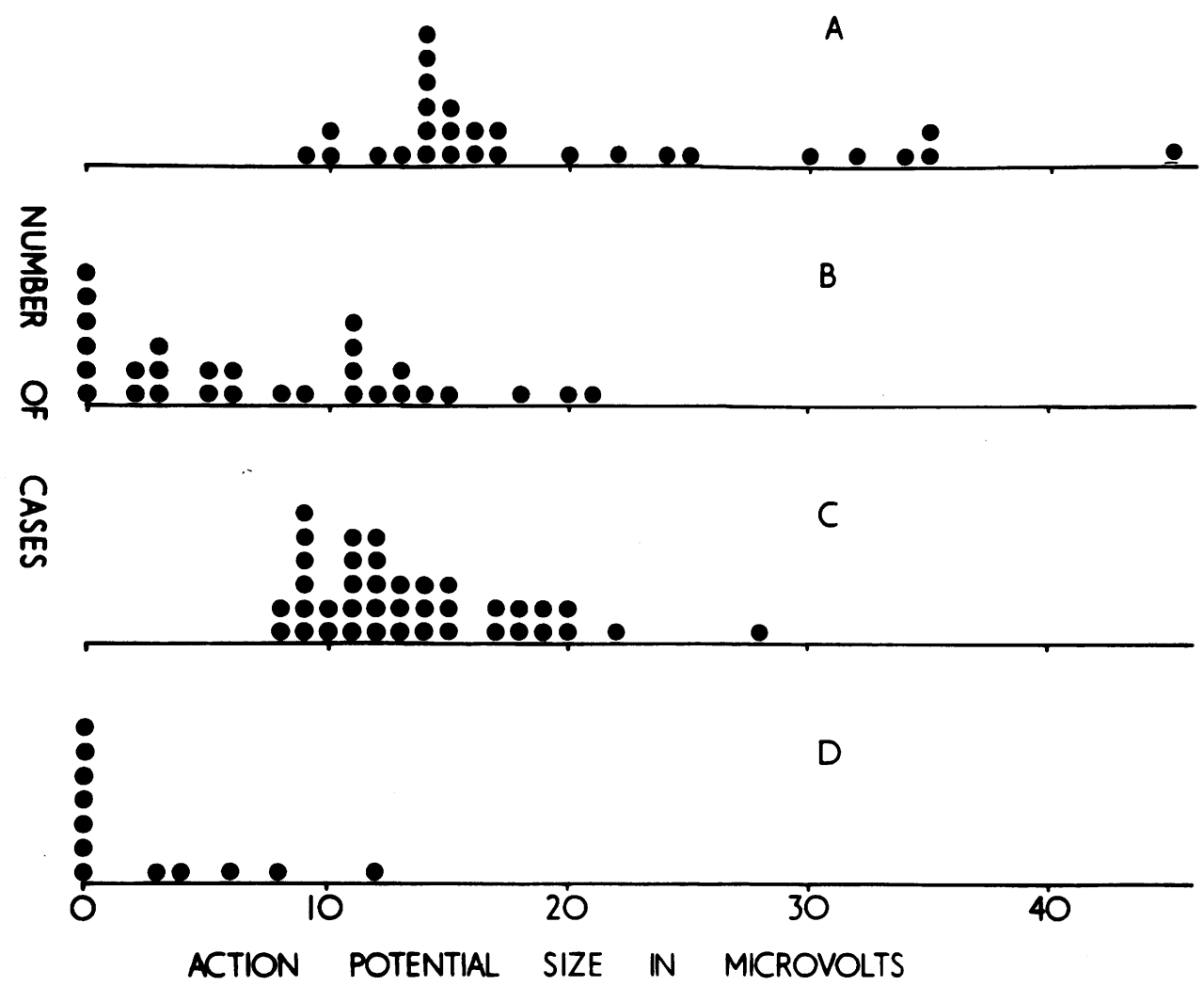

Fig. 4. - Sensory action potential amplitude in different individuals. All potentials recorded at the wrist.

(A) From the median nerve during stimulation of the index finger in control subjects.

$(B)$ The same in patients with suspected median nerve lesions.
$(C)$ From the ulnar nerve during stimulation of the little finger in control subjects.

(D) The same in patients with suspected ulnar nerve lesions. clinical findings is prevented by the difficulty of expressing minor degrees of sensory loss in an objective or quantitative manner, but in general there is no doubt that the nerve action potential was most likely to be abnormal in patients who showed obvious sensory defects. For example, there were 15 patients with suspected median nerve lesions in whom sensation was normal on clinical testing; the sensory action potential was absent in one of them and less than 5 microvolts in amplitude in another. In eight patients with some impairment of superficial sensation on the fingers but without any defect of two-point discrimination there were two with absent nerve action potentials, and two others with potentials which were less than 5 microvolts in amplitude. There were six patients with defective two-point discrimination on the fingers in addition to superficial sensory impairment to pin-prick and cotton wool, and nerve action potentials were absent in three of them; in the remaining three patients recorded potentials were all less than 5 microvolts in amplitude.

In the patients without sensory loss, action potential latencies of less than 4 milliseconds were recorded in seven cases. In patients with some clinical sensory disturbance but without defects of two-point discrimination, latencies of less than 4 milliseconds were found in two cases. In the group of patients with two-point loss, a latency of less than 4 milliseconds was found in only one case.

Recovery of Nerve Action Potential after Operation on Carpal Tunnel.-Repeated observations on patients after operation were possible in 10 cases, and in all of them some recovery of the nerve action potential was seen. These serial observations were not continued for as long as we would have liked, as few patients were prepared to attend for tests on more than one or two occasions after their symptoms had disappeared, and in general the patients' symptoms seemed to resolve a considerable 


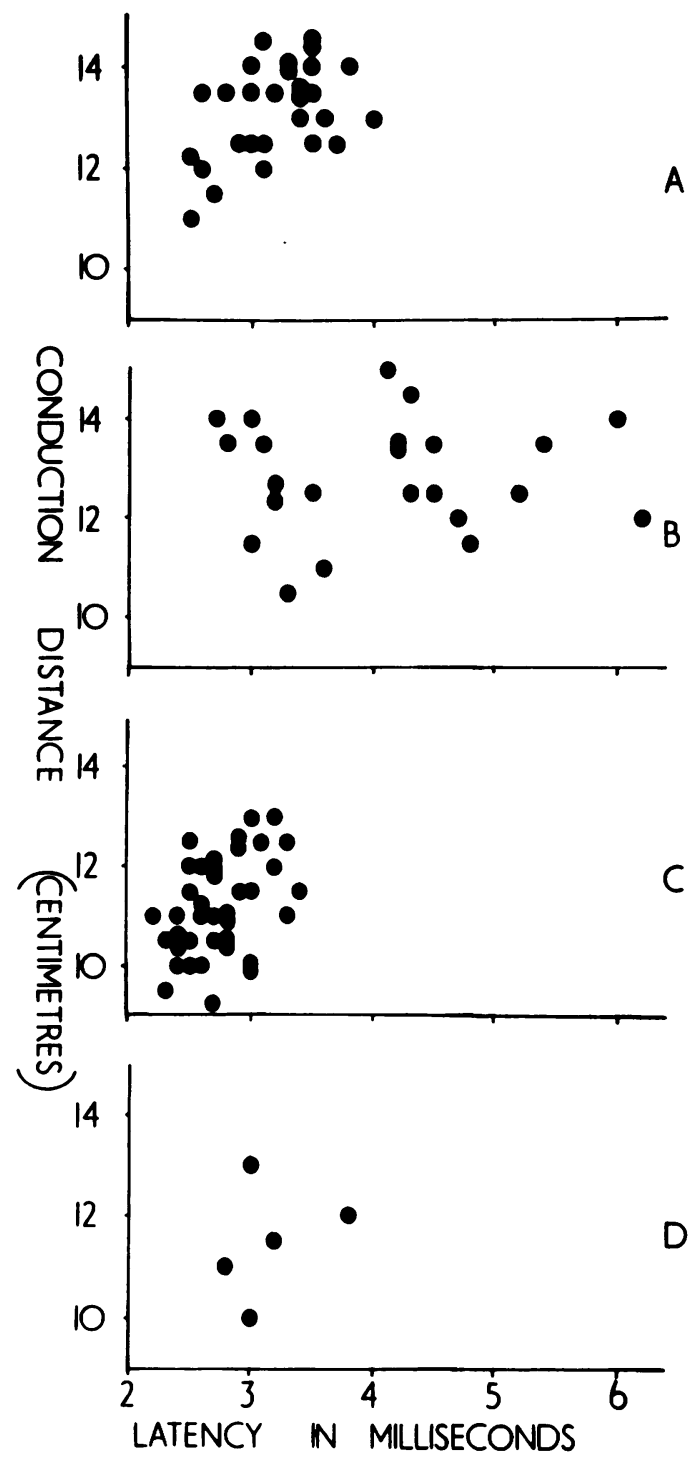

FIG. 5.-Sensory action potential latency in different individuals plotted against conduction distance (stimulating cathode to wrist electrode) in each case. Groups $A, B, C$, and $D$ as in Fig. 4.

time before the nerve action potential became normal. These points are illustrated by the following case history.

Mrs. C. B., a 48-year-old housewife, was admitted to the National Hospital in May, 1956, with the complaint of pain and numbness in the right arm during the preceding year. She was awakened at night by numbness of the right hand and forearm accompanied by aching pain deep in the palm and in the muscles of the forearm, these symptoms lasting for up to an hour. After a few months she developed tingling sensations in the right thumb, index and middle fingers; at first these occurred only at night but later they persisted throughout the day, being made worse by using the hand. Examination on admission revealed weakness of the abductor pollicis brevis and subjective sensory impairment to pin-prick and cotton wool over the thumb, index and middle fingers, and over the median-supplied half of the ring finger. Appreciation of joint movement was intact in the digits, but on the pad of the index finger the patient made errors in discrimination of compass points $0.5 \mathrm{~cm}$. apart; there were no errors at $0.75 \mathrm{~cm}$. separation on the index finger or at $0.5 \mathrm{~cm}$. On the little finger.

Sensory action potentials were examined with ring stimulating electrodes round the index finger and surface recording electrodes over the median nerve trunk just above the wrist (conduction distance, $13.5 \mathrm{~cm}$.). A small action potential, approximately 3 microvolts in amplitude, was recorded which could not be made larger by increasing the intensity of the stimulus. Action potential latency measured from the stimulus artefact to the peak of the main upward deflection was 5.4 milliseconds (Fig. 6A).

The right flexor retinaculum was divided by Mr. J. Hankinson, and clinical improvement was rapid. When seen on the 27th post-operative day, the patient was symptom free and no sensory deficit could be detected in the right hand. The electrical test was repeated with the same electrode positions and stimulating voltage as before, and it showed a sensory action potential with an amplitude of 11 microvolts and a latency of 4.1 milliseconds (Fig. 6B). Although sensation appeared clinically to have returned to normal within a month of operation,
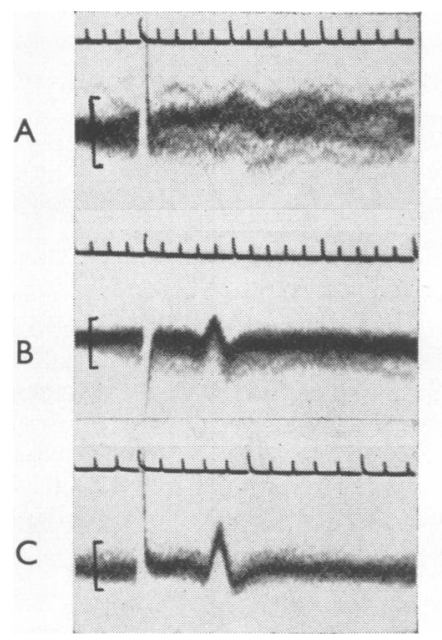

PRE-OPERATIVE

27 TH POST-OP. DAY

92 ND POST-OP. DAY

Fig. 6.-Mrs. C. B.: carpal tunnel syndrome.

Recordings from the median nerve at the wrist during stimulation of the index finger before and after division of the flexor retinaculum. Tracings show stimulus artefact followed by nerve action potential in each case.

Calibration $20 \mu \mathrm{V}$. Time scale 1 and 5 milliseconds; 20 sweeps superimposed. 
a third electrical test was carried out on the 92nd postoperative day (Fig. 6C) which showed further improvement in both action potential size (19 microvolts) and latency (3.7 milliseconds).

At the time of each test, control observations were made on the ulnar nerve at the wrist during stimulation of the little finger. Sensory action potentials recorded from the ulnar nerve were not significantly different in the pre- and post-operative periods, the figures for action potential amplitude being successively $10.8,11.0$, and 10.5 microvolts. On all three occasions ulnar action potential latency was between 2.6 and 2.8 milliseconds.

Ulnar Nerve Lesions at the Elbow.-Twelve patients with suspected ulnar nerve lesions at the elbow were examined. Wasting of the ulnar-supplied muscles was present in nine patients and weakness without wasting in two others. Subjective impairment of superficial sensation in the ulnar territory was present in 11 patients and discrimination of compass points on the little finger was defective in six of them.

Sensory nerve action potentials were examined with stimulating electrodes round the little finger and recording electrodes over the ulnar nerve trunk just above the wrist. Stimulus intensity was kept as high as could be tolerated by each individual but, in spite of this, nerve action potentials were absent in seven cases and of small amplitude in several others (Fig. 4).

Action potential latency could only be measured in five patients and in none of them was it obviously abnormal (Fig. 5). In one patient, however, a small increase in latency was thought to be present as shown by comparison between the affected hand and the ulnar nerve on the opposite side.

The patient, a 50-year-old housewife, showed mild weakness of the ulnar-supplied muscles on one side with subjective impairment of superficial sensation over the little finger and half of the ring finger, but with preservation of two-point discrimination. When the little finger on the affected side was stimulated, an action potential 8 microvolts in amplitude was recorded from the wrist, its latency being 3.8 milliseconds (conduction distance, $12 \mathrm{~cm}$.). When the little finger on the opposite side was stimulated in the same way, the ulnar action potential at the wrist was 11 microvolts in amplitude with a latency of only 2.7 milliseconds although the conduction distance was the same $(12 \mathrm{~cm}$.).

When patients with suspected ulnar nerve lesions showed a sensory action potential at the wrist which was only doubtfully reduced in size, more information was sometimes obtained by stimulating the nerve trunk at the wrist, and recording the mixed sensory and antidromic motor volley from the ulnar nerve above the elbow. The findings in one such case are described in detail below.
Mr. G. S., aged 56, was referred to one of us in October, 1956, with a history of numbness and tingling in the fourth and fifth fingers of the left hand for four weeks. In the past, it had not been uncommon for him to wake in the mornings with transient numbness of the ulnar fingers, but in September, 1956, he had awakened with numbness which did not disappear as it had done before. Examination four weeks after the onset showed slight weakness of the ulnar-supplied muscles in the left hand without wasting. Power in the flexor carpi ulnaris seemed normal. There was a subjective change in the sensation aroused by a firm touch on the one and a half fingers supplied by the ulnar nerve and on the ulnar side of the hand, without real impairment of appreciation of pin-prick or cotton wool. There were, however, occasional errors in discrimination of compass points $0.5 \mathrm{~cm}$. apart on the fifth finger which were not present elsewhere. The ulnar nerve itself was not thickened at the elbow.

When the left abductor digiti minimi was sampled with a concentric needle electrode, the electromyograph showed no abnormal insertion activity or spontaneous fibrillation, and the number of motor units under voluntary control was not obviously reduced. Conduction velocity in the motor fibres of the left median and ulnar nerves was measured with stimulating electrodes placed just above the wrist and elbow, and recording electrodes (concentric needles) in the abductor pollicis brevis and the abductor digiti minimi respectively. There was no difference in conduction rate between the two nerves distal to the wrist, but in the segment which included the elbow region, calculated conduction velocity for the fastest motor fibres in the ulnar nerve was 35 metress per second as opposed to $\mathbf{5 2}$ metres per second for the median nerve.

Sensory action potentials were examined with ring stimulating electrodes round the index and little fingers and surface recording electrodes over the median and ulnar nerve trunks just above the wrist (Fig. 7). The ulnar action potential was rather small (amplitude 6 microvolts) compared with the median (amplitude 18 microvolts), but was not considered to be clearly abnormal. The median and ulnar nerve trunks were then stimulated at wrist level, with recording electrodes above the elbow. A normal afferent volley was recorded without difficulty from the median nerve in this way, but from the ulnar nerve a small delayed action potential was obtained which could not be made larger by increasing stimulus intensity.

Brachial Plexus Lesions.-Two patients were examined with severe sensory loss in the hand due to a lesion of the brachial plexus. In both cases the brachial plexus on one side was invaded by malignant deposits, the site and nature of the lesion being confirmed by exploration and biopsy. In neither case could a sensory nerve action potential be detected at the wrist when the affected fingers were stimulated whereas normal potentials were obtained without difficulty in the opposite arm. In one patient with invasion of the lower trunk of the brachial 

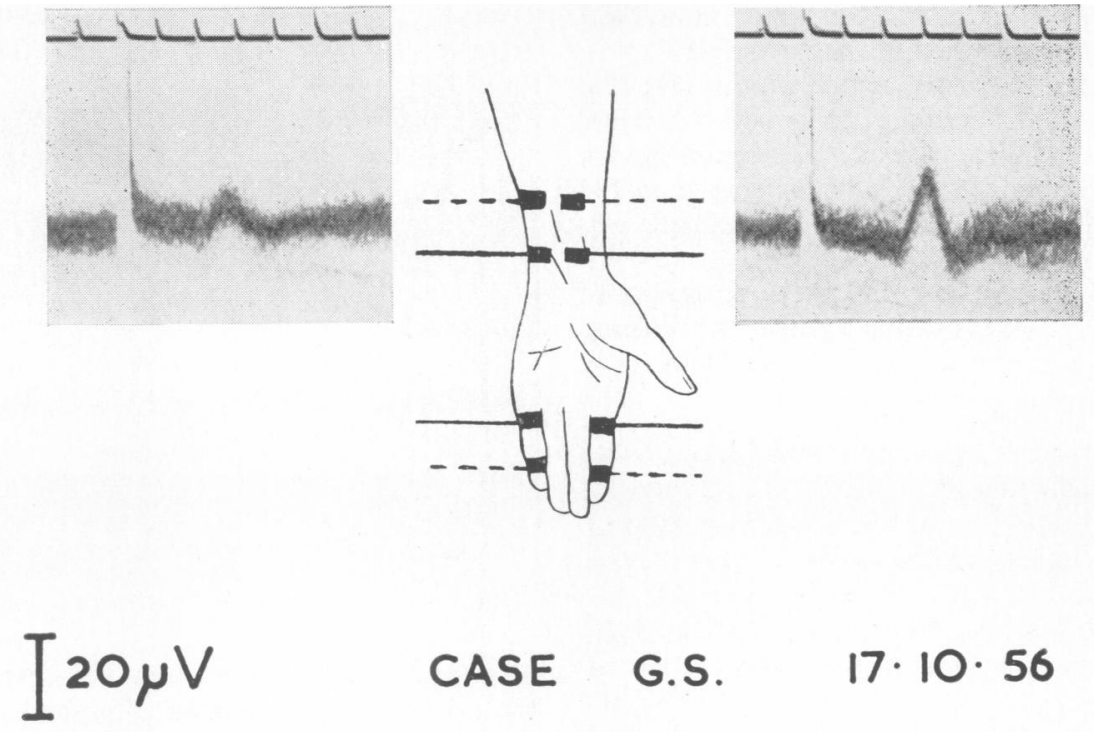

CASE G.S.

$17 \cdot 10 \cdot 56$
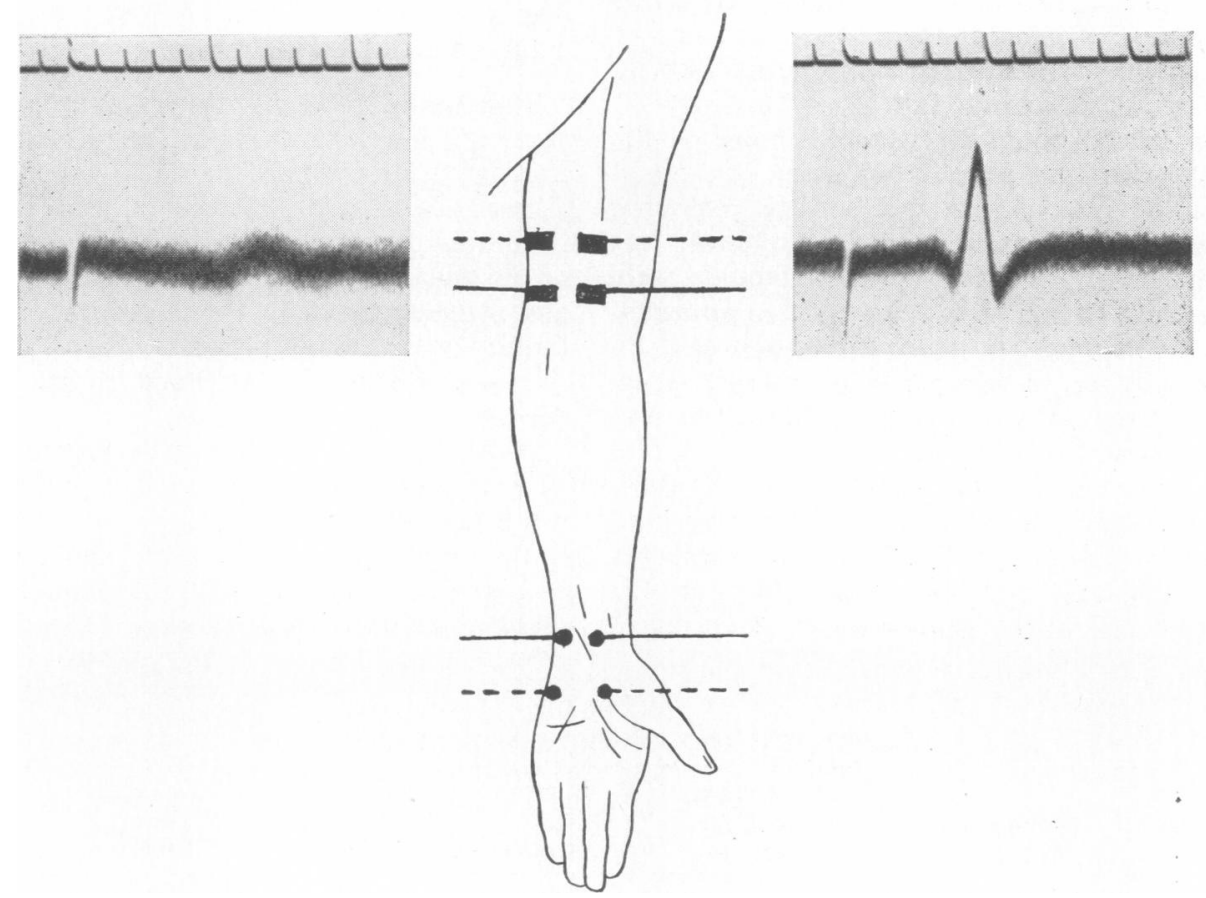

Fig. 7.-Mr. G. S.: ulnar nerve lesion at the elbow. Lower tracings show records from median and ulnar nerves just above the elbow during supramaximal stimulation of nerve trunks at the wrist. Upper tracings show sensory action potentials recorded at the wrist during stimulation of fingers. Time scale 1 and 5 milliseconds; 20 sweeps superimposed.

plexus by mammary carcinoma, sensory loss was confined to the inner side of the arm and hand and to the ring and little fingers, with sparing of the thumb and index finger and only doubtful subjective diminution in superficial sensation over the middle finger. In the little finger itself sensory loss 
was severe, with almost complete superficial anaesthesia and considerable proprioceptive loss. In this patient no sensory nerve action potential could be detected in the ulnar nerve when the little finger was stimulated whereas a normal action potential (amplitude 15 microvolts) was recorded from the median nerve when the index finger was stimulated.

These findings serve to confirm the reliability of the technique in demonstrating changes in the distal part of a sensory nerve when the causal lesion is as high as the brachial plexus.

Polyneuritis.-Sensory nerve conduction was examined in seven patients with chronic polyneuritis of varying aetiology. All these patients showed symmetrical peripheral weakness with loss of tendon jerks and in all but one of them sensory loss was present in both hands and feet. In Table I a simple method of grading the motor and sensory signs in the hands has been used, which shows that the severity of involvement varied widely in different patients. All the patients were seen more than two months after the onset of their illness and one of them (Case E. W.) had made a substantial clinical recovery by the time of the test.

Sensory action potentials were examined in the usual way with ring stimulating electrodes round the index or little finger and surface recording electrodes over the appropriate nerve trunk just above the wrist. Sensory action potentials were absent in five patients although sensation appeared clinically to be perfectly preserved in one of them and to be only slightly affected in two others. In the remaining two patients small delayed sensory potentials were recorded (Fig. 8).

Motor nerve conduction velocity in the forearm was also examined in each case, stimulating electrodes being placed over the ulnar or median nerve trunk just above the wrist and elbow, with recording needle electrodes in the small muscles of the hand.

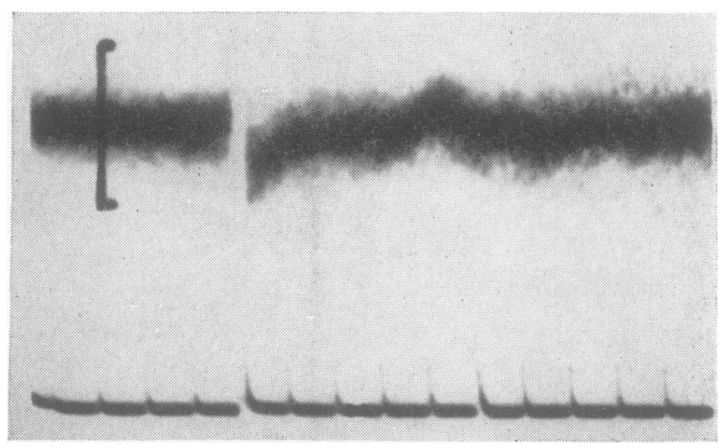

FIG. 8.-Mr. H. S.: progressive polyneuritis with retinitis pigmentosa and deafness. Clinical diagnosis Refsum's disease. Record from median nerve at the wrist during stimulation of index finger shows stimulus artefact followed by small delayed nerve action potential (conduction distance $12.5 \mathrm{~cm}$.). Calibration $20 \mu \mathrm{V}$. Time scale 1 and 5 milliseconds; 20 sweeps superimposed.

Slowing of motor nerve conduction, which is known to occur in polyneuritis (Lambert, 1956), was present in all seven of our patients (Table I), the degree of slowing showing little relation to the clinical state of muscle power.

Peroneal Muscular Atrophy.-Two patients with peroneal muscular atrophy were examined, the findings being included in Table $I$. Both patients showed the classical picture of pes cavus accompanied by slowly progressive wasting and weaknese of the lower limbs, starting in their peripheral parts and spreading later to the thighs and to the upper limbs. In the case of patient L. S., an affected relative was examined and found to have a similar condition.

From Table I it can be seen that no sensory action potentials were recorded in either patient, and that marked slowing of motor nerve conduction velocity in the forearm was also present. The motor findings in Case G. M. have already been described in detail elsewhere (Gilliatt and Thomas, 1957).

TABLE I

POLYNEURITIS AND PERONEAL MUSCULAR ATROPHY

\begin{tabular}{|c|c|c|c|c|c|c|}
\hline Case & Aetiology & $\begin{array}{l}\text { Paralysis } \\
\text { of } \\
\text { Intrinsic } \\
\text { Muscles }\end{array}$ & $\begin{array}{l}\text { Sensory } \\
\text { Loss } \\
\text { in } \\
\text { Fingers }\end{array}$ & $\begin{array}{c}\text { Motor Nerve } \\
\text { Conduction Velocity } \\
\text { in Forearm** } \\
\text { (metres/sec.) }\end{array}$ & $\begin{array}{c}\text { Sensory } \\
\text { Action } \\
\text { Potentials }\end{array}$ & $\begin{array}{c}\text { Nerve } \\
\text { Examined }\end{array}$ \\
\hline $\begin{array}{l}\text { J. R. } \\
\text { A. F. } \\
\text { E. S. } \\
\text { W. F. } \\
\text { R. H. } \\
\text { H. S. } \\
\text { E. W. } \\
\text { G. M. } \\
\text { L. S. }\end{array}$ & $\begin{array}{l}\text { Unknown } \\
\text { Unknown } \\
\text { Polyarteritis nodosa } \\
\text { Unknown } \\
\text { Familial, hypertrophic } \\
\text { Refsum's disease** } \\
\text { Familial, hypertrophic } \\
\text { Peroneal muscular atrophy } \\
\text { Peroneal muscular atrophy }\end{array}$ & $\begin{array}{c}+++ \\
+++ \\
\text { Complete } \\
+ \\
++ \\
++ \\
+ \\
+++ \\
++\end{array}$ & $\begin{array}{c}+ \\
+++ \\
+++ \\
+ \\
++ \\
++ \\
\text { No sensory loss } \\
+ \\
+\end{array}$ & $\begin{array}{c}25 \cdot 9 \\
7 \cdot 1 \\
\text { Nerve inexcitable } \\
17 \cdot 0 \\
6 \cdot 8 \\
38 \cdot 5 \\
14 \cdot 4 \\
11 \cdot 2 \\
26 \cdot 4\end{array}$ & $\begin{array}{l}\text { Small delayed } \\
\text { Absent } \\
\text { Absent } \\
\text { Absent } \\
\text { Absent } \\
\text { Small delayed } \\
\text { Absent } \\
\text { Absent } \\
\text { Absent }\end{array}$ & $\begin{array}{l}\text { Median } \\
\text { Median } \\
\text { Median } \\
\text { Ulnar } \\
\text { Ulnar } \\
\text { Median } \\
\text { Ulnar } \\
\text { Ulnar } \\
\text { Median }\end{array}$ \\
\hline
\end{tabular}

* Clinical details of this patient are contained in a paper to be published by Dr. N. S. Gordon. 


\section{Discussion}

Our results have confirmed Dawson's original observation that a small action potential can be recorded from the median or the ulnar nerve trunk at the wrist when brief electrical shocks are applied to the digital nerves in the fingers. In our study nerve action potentials appeared so constantly in control subjects that their absence has been regarded as a reliable sign of disturbed function. However, the potentials recorded from healthy nerves varied considerably in amplitude in different individuals, so that little significance has been attached to small variations in action potential size in pathological material. In doubtful cases, measurement of action potential latency was often helpful, particularly when a damaged nerve could be compared with an unaffected nerve in the same or the opposite arm. Latency measurements were most valuable in the group of patients with median neuritis at the wrist, values in excess of the longest control figure being found in more than half of them. This result may be compared with the marked slowing of motor nerve conduction which has been shown to occur in this condition (Simpson, 1956).

In attempting to relate clinical and electrical findings there are a number of difficulties. In the first place the sensory fibres contributing to the nerve action potential are believed to be large myelinated fibres, since their conduction velocity between wrist and elbow is at least as great as the maximal conduction velocity of motor nerve fibres supplying the small muscles of the hand (Dawson, 1956). Therefore these potentials provide no information about the small myelinated and nonmyelinated fibres which are thought to be concerned with pain and temperature sensation. Of the large myelinated fibres contributing to the nerve action potential, some may be presumed to convey impulses from the skin whereas others supply deep structures such as joint capsules and periosteum. It must therefore be concluded that the nerve action potential does not provide direct information about the functional state of any single sensory modality.

In relating electrical and clinical findings there is a further complication which derives from the nature of the stimulus. To produce synchronous volleys we have used an electrical shock which is sufficient in a normal nerve to excite a large number of fibres. A damaged fibre, however, may still be capable of responding to physiological stimuli through its endorgan when its electrical threshold is so high that it no longer responds to a brief shock applied to the nerve trunk. This would have the effect of reducing or abolishing the potential that we attempt to record without exerting a comparable effect on sensory function.
The nerve action potential that we have recorded is formed from the summed potentials of a large number of nerve fibres, and its appearance depends upon the passage of a synchronous volley under the recording electrodes. Slowing of nerve conduction has the effect of scattering the time of arrival of different impulses at the recording electrodes and thus of reducing the peak amplitude of the compound action potential. Slowing might therefore be expected to produce more disturbance of the nerve action potential than of sensory function, and there is some support for this in our records. In the patients with polyneuritis, motor nerve conduction velocity was also measured and was often slowed to less than half the normal figure. In these patients the sensory nerve action potential was almost always absent, and yet some of them showed little or no deficit on formal sensory testing. This sort of result is most easily explained by the assumption that nerve conduction was grossly slowed in all large myelinated fibres, but lost completely in very few of them.

The site of the nerve lesion is of obvious importance in considering the relation between clinical and electrical findings. In patients with median neuritis at the wrist, the segment of nerve examined has always included the damaged portion, a fact which has enabled a high proportion of mild cases to be recognized. With ulnar nerve lesions at the elbow recording of sensory potentials at the wrist can only be expected to show changes if a proportion of fibres undergo degeneration in their distal parts. In mild ulnar nerve lesions more striking abnormalities may be demonstrated by stimulating the ulnar nerve trunk at the wrist and recording the mixed sensory and motor nerve action potential above the elbow. In the case of brachial plexus lesions we have not succeeded in devising any method of recording above the level of the lesion and have been forced to restrict ourselves to the observation of changes in the distal parts of the sensory nerves.

From what has been said above, it is clearly impossible to equate electrical and clinical findings in patients except in the most general terms. The relationship between electrical and clinical abnormalities varies not only in different pathological conditions but also in different individuals with the same condition, and even in the same person at different times, e.g., before and after operation on the carpal tunnel. In general, however, it may be said that patients with severe sensory loss in the hand have all shown abnormalities of the nerve action potential whereas the reverse has not been true, several patients with abnormal or absent nerve potentials having been without demonstrable sensory loss. 
As a diagnostic procedure, the method has the great advantage that it provides information about the function of sensory nerve fibres which is objective and obtained directly from the nerve, without any cooperation from the patient except insofar as he is required to lie still and relax during the test. From patients who are unable to cooperate at all the information can still be obtained by examination under anaesthesia. It must be accepted that the technical difficulties involved in recording nerve action potentials are greater than those encountered in ordinary electromyography. The potentials to be recorded are much smaller, and therefore require higher amplification and more careful technique. With practice, however, the procedure is not more time-consuming than other electro-diagnostic tests, and if suitable apparatus is available there is nothing inherently impossible in its use as a routine diagnostic procedure.

\section{Summary}

The digital nerves in the fingers have been stimulated electrically with single shocks and the action potential of the afferent volley recorded through surface electrodes placed over the median and ulnar nerve trunks just above the wrist.

Sensory action potentials from the fingers were recorded from a large number of healthy median and ulnar nerves. Sixty-seven control observations were made, an action potential being recorded successfully in every case.
Forty-one patients with suspected median and ulnar nerve lesions were examined. In 13 patients, no sensory action potential could be recorded from the affected nerve when the appropriate finger was stimulated. In other patients, the recorded action potentials were of small amplitude and long latency when compared with the control group.

Two patients had brachial plexus lesions causing sensory loss in the hand. In neither of them could a sensory action potential be recorded from the wrist when an affected finger was stimulated.

Seven patients with chronic polyneuritis and two with peroneal muscular atrophy were examined. Sensory action potentials were small and delayed in two cases and absent in the remainder.

It is concluded that the technique can be of practical value in the investigation of patients with sensory disorders affecting the upper limbs.

We wish to thank members of the staff of the National Hospital who have referred patients for investigation. We also wish to thank Dr. G. D. Dawson and Dr. W. A. Cobb for helpful criticism and advice, and Mr. H. B. Morton for technical assistance.

\section{REFERENCES}

Dawson, G. D. (1947). J. Neurol. Neurosurg. Psychiat., 10, 137. - (1956). J. Physiol. (Lond.), 131, 436. , and Scott.

Gilliatt, R. W. and Thomas, P. K (1957). Ann. phys Med. 4, 10 Lambert, E. H. (1956). In Clinical Examinations in Neurolog [Mayo Clinic]. Saunders, Philadelphia.

Simpson, J. A. (1956). J. Neurol. Neurosurg. Psychiat., 19, 275. Wagman, I. H., and Lesse, H. (1952). J. Neurophysiol., 15, 235. 